\title{
Giant Ectopic Parathyroid Adenoma of the Mediastinum Caus- ing Primary Hyperparathyroidism - A Rare Case Report with Review of Literature
}

\author{
Swetha Narla ${ }^{1 *}$, Sasankh $\mathrm{RK}^{2}$, Annapurneswari $S^{3}$ and Chandrasekaran Maharajan ${ }^{4}$ \\ ${ }^{1}$ Consultant, Department of Histopathology, Apollo Cancer Centre, India \\ ${ }^{2}$ Senior Consultant, Department of Cardiothoracic surgery, Apollo Cancer Centre, India \\ ${ }^{3}$ Senior Consultant, Department of Histopathology, Apollo Cancer Centre, India \\ ${ }^{4}$ Senior Consultant, Department of Endocrine surgery, Apollo Cancer Centre, India
}

*Corresponding author: Dr. Swetha Narla, Consultant, Department of Histopathology, Apollo Cancer Centre, 320, D block, Anna Salai, Chennai, India, Tel: 09884087859

\begin{abstract}
Primary hyperparathyroidism is characterized by high parathyroid hormone levels and calcium levels which is most commonly due to a parathyroid adenoma. Ectopic parathyroid adenoma is very rare and is the cause for failed primary surgery. Common sites include mediastinum, path of vagus nerve, recurrent laryngeal nerve and intrathyroidal locations. Combination of imaging techniques including Sestamibi scan is very useful in locating an ectopic parathyroid adenoma and helps to avoid re explorative surgery for recurrence. Here we present a case report of a 39-year-old woman who was referred to us for generalized body ache. On work up, was diagnosed with primary hyperparathyroidism due to a giant ectopic parathyroid adenoma which was confirmed on histopathological examination. Though rare, ectopic parathyroid adenoma should always be considered whenever a diagnosis of primary hyperparathyroidism is made.
\end{abstract}

\section{Keywords}

Adenoma, Calcium, Ectopic, Mediastinum, Parathyroid, Sestamibi scan

\section{Introduction}

Primary hyperparathyroidism is one of the common endocrinopathies with an annual incidence of 0.1$3 \%,[1,2]$ most of which is due to a solitary adenoma. Frame and Jackson [3] have described the symptoms of hyperparathyroidism in a triad of bones, stones and abdominal groans. Parathyroid glands originate from the endoderm of third and fourth pharyngeal pouches and can be found in any location of its descent from the pouch till the mediastinum. Common sites include mediastinum, path of vagus nerve, recurrent laryngeal nerve and intrathyroidal location [4]. Ectopic parathyroid adenoma is very rare and is one of the causes for failed primary surgery. We present a case of Giant ectopic parathyroid adenoma in the mediastinum.

\section{Case Report}

A 39-year-old woman was referred to us for generalized body ache of two months duration. She did not have any significant past medical or surgical history. Physical examination showed no abnormality. Routine investigations revealed elevated serum calcium levels $11.2 \mathrm{mg} / \mathrm{dL}$ (normal range $8.5-10.2 \mathrm{mg} / \mathrm{dl}$ ). Thyroid function test was normal. Other blood investigations were normal. As a part of workup for hypercalcemia, serum parathyroid hormone (PTH) level was checked and it was elevated $-222.3 \mathrm{pg} / \mathrm{ml}$ (normal range $8-51 \mathrm{pg} /$ $\mathrm{ml}$ ). Serum magnesium was $2.33 \mathrm{mg} / \mathrm{dl}$ (normal range $1.5-2.5 \mathrm{mg} / \mathrm{dl}$ ) and Vitamin D was $20 \mathrm{ng} / \mathrm{ml}$ (normal range $10-20 \mathrm{ng} / \mathrm{ml}$ ). In view of raised serum PTH level, Sestamibi scan was done which revealed an intrathoracic ectopic parathyroid adenoma in addition to left inferior parathyroid adenoma. CT scan of neck and chest

\footnotetext{
Citation: Narla S, Sasankh RK, Annapurneswari S, Maharajan C (2020) Giant Ectopic Parathyroid Adenoma of the Mediastinum Causing Primary Hyperparathyroidism - A Rare Case Report with Review of Literature. Int J Pathol Clin Res 6:109. doi.org/10.23937/2469-5807/1510109

Accepted: April 29, 2020: Published: May 01, 2020

Copyright: (c) 2020 Narla S, et al. This is an open-access article distributed under the terms of the Creative Commons Attribution License, which permits unrestricted use, distribution, and reproduction in any medium, provided the original author and source are credited.
} 


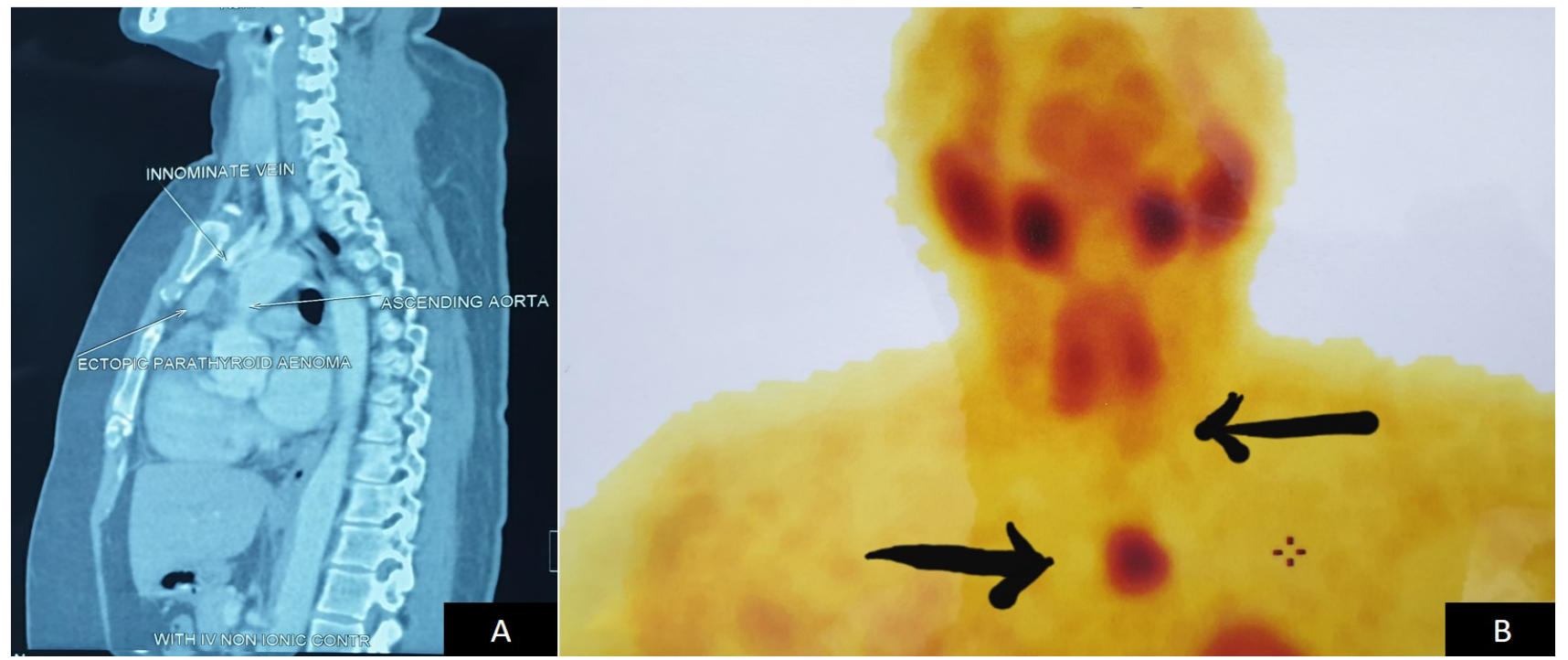

Figure 1: A) CT scan showing an III-defined enhancing lesion in the anterior mediastinum in pre vascular space beneath the sternomanibrium joint level inferior to innominate vein and anterior to ascending aorta; B) Sestamibi scan was suggestive of an intrathoracic ectopic parathyroid adenoma along with a left inferior parathyroid adenoma.

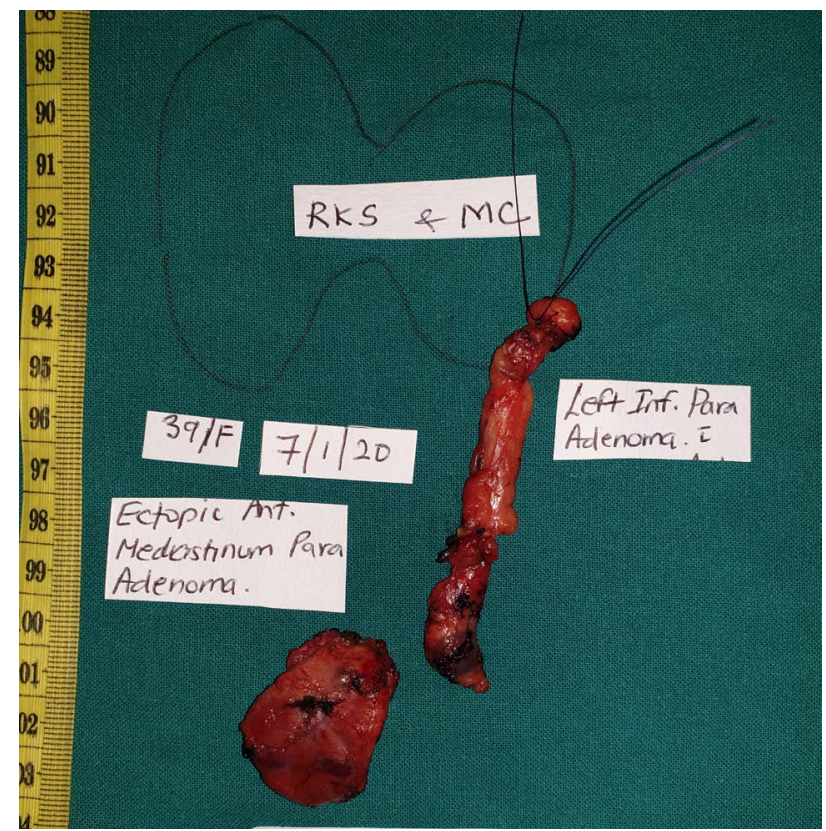

Figure 2: Specimen photograph of Ectopic parathyroid adenoma, Left inferior parathyroid adenoma and the connecting track.

with contrast showed a left inferior parathyroid adenoma and an ill-defined enhancing lesion in the anterior mediastinum in pre vascular space measuring $2.6 \times 2.5 \times$ $1.2 \mathrm{~cm}$, lesion was beneath the sternomanubrium joint level inferior to innominate vein and anterior to ascending aorta. In order to confirm, Tc99mm Sestamibi SPECT (Single Photon Emission Computed Tomography) was performed which was suggestive of ectopic parathyroid adenoma in intrathoracic region and left inferior parathyroid adenoma (Figure 1). Patient was admitted for excision of intrathoracic ectopic parathyroid adenoma and left inferior parathyroid adenoma. Under general anaesthesia, surgery was performed in 2 steps. Through a skin collar crease, incision skin flaps were raised. Ster- nothyroid and sternohyoid muscles were retracted. Excision of left inferior parathyroid adenoma was carried out after identifying and preserving the left recurrent laryngeal nerve. The other three parathyroid glands were explored and they all appeared normal. Since the mediastinal adenoma was inferior to the Innominate vein and in front of the ascending aorta, excision of the same was not attempted through the cervical incision. Anterior mediastinal adenoma was exposed via median sternotomy using a midline incision extending from the suprasternal notch to the xiphoid process. Mediastinum was entered after cutting the sternum using a pneumatic sternal saw. There was a circumscribed adenoma seen inferior to the innominate vein and in front of ascending aorta which was excised in toto along with the tract connecting it to the left inferior parathyroid adenoma and were sent for histopathological examination (Figure 2). $12 \mathrm{hrs}$ after surgery, serum PTH level dropped to $13.8 \mathrm{pg} / \mathrm{ml}$ and $24 \mathrm{hrs}$ after surgery, serum calcium level dropped down to $8.1 \mathrm{mg} / \mathrm{dL}$. Patient had developed symptoms of hypocalcemia in the post-operative period and was treated with intravenous calcium gluconate.

Ectopic parathyroid adenoma measured $3.8 \times 2.3 \times$ $1.5 \mathrm{~cm}$ and weighed $6 \mathrm{gms}$. The external surface was smooth. Cut surface showed diffuse solid grey brown areas. The left inferior parathyroid adenoma measured $1 \times 1 \times 0.8 \mathrm{~cm}$ and weighed $1 \mathrm{gm}$. Cut surface showed grey brown areas. There was a tract connecting both adenomas. Histopathological examination of the mediastinal adenoma showed encapsulation with thymic tissue adjacent into it. The lesion was composed of clear cells arranged in an organoid and follicular pattern separated by thin fibrous septae. The cells were polygonal with moderate cytoplasm, well-defined cell borders and inconspicuous nucleoli. There was no evidence of capsular/vascular/lymphatic invasion. The left inferior parathyroid adenoma showed similar histomorphology with 


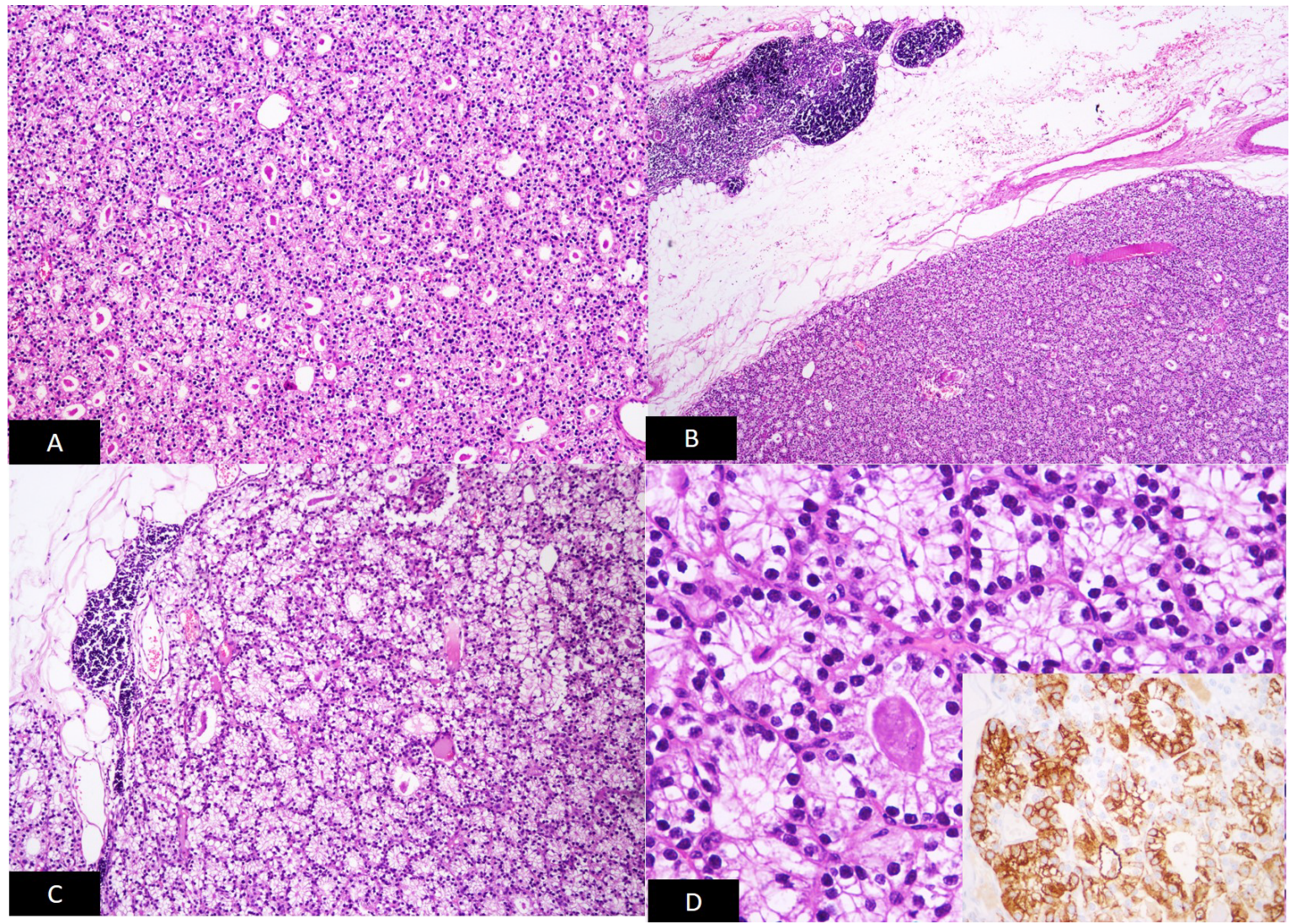

Figure 3: A) Follicular pattern of arrangement of tumor cells in the left inferior parathyroid gland (H \& $E$ 100x and 400x); B), C) and D) Similar appearing cells in the mediastinum adenoma with peripherally compressed thymic tissue (H \& $E$ 100x and 400x).

Inset showing tumor cells staining diffusely with Chromogranin immunostain.

compressed normal parathyroid tissue at the periphery. Immunohistochemical confirmation with Chromogranin antibody showed diffuse staining within the tumor cells (Figure 3). The tract connecting both the adenomas also showed scant foci of parathyroid tissue with no specific pathology. The differential diagnosis considered on histomorphology were that of a parathyroid adenoma, thyroid neoplasm and metastatic adenocarcinoma with clear cell pattern. The tumor cells had clear cytoplasm with well-defined cell borders and intraluminal secretions resembling colloid. Raised serum parathyroid hormone and calcium levels, reduction in serum PTH and calcium levels post-surgery, confirmed the diagnosis of parathyroid adenoma, excluding thyroid neoplasm and metastasis. Since the other three parathyroid glands were normal, parathyroid hyperplasia was excluded.

\section{Discussion}

Hyperparathyroidism can be due to primary, secondary and tertiary causes. Primary hyperparathyroidism is characterized by high parathyroid and calcium levels which is most commonly due to a parathyroid adenoma $(90 \%)$ and due to multiple adenomas, hyperplasia or carcinoma in $10 \%$ of patients [5]. Mean age of presentation of parathyroid adenoma is 60 years and is common in women (90\%). Symptoms of hypercalcemia range from osteoporosis, vomiting, constipation, calculus, polyuria, polydipsia, etc. Prevalence of pathologic crisis varies from $6-30 \%$. Acute pancreatitis, kidney damage and involvement of the bone are major long-term complications of primary hyperparathyroidism. Involvement of bone can be in the form of generalized demineralization, focal lytic lesion or subperiosteal resorption. Osteitis fibrosa cystica is end stage of bone remodeling in chronic hyperparathyroidism. Ectopic parathyroid adenomas have increased risk of bone involvement compared to others [6]. Most parathyroid adenomas are less than $1 \mathrm{gm}$. Ectopic parathyroid adenomas range from 2 to 3 gms and are rare [7]. Parathyroid neoplasms can be associated with MEN type I which include primary hyperparathyroidism, pituitary adenoma and gastric neuroendocrine tumors [8]. Syndromic workup needs to be done before considering an isolated parathyroid adenoma. Sulaiman, et al. showed that ectopic parathyroid adenomas can have chromosomal abnormalities/ have genetic mutations [9].

Churchill in 1932, first reported a case of mediastinal ectopic parathyroid adenoma [10]. There are two superior and two inferior parathyroid glands. Superior parathyroid glands are derived from fourth pharyngeal pouch and inferior parathyroid glands develop from dorsal wing of third pharyngeal pouch [11]. Thymic gland comes from the ventral wing of third pharyngeal pouch. Since the descending route of inferior parathy- 
roid glands is same as thymus, ectopic location in the mediastinum is known to occur [11]. Most common ectopic locations in descending order include paraoesophageal region $(45 \%)$, cervical thymus, $(25 \%)$, cervical mediastinum (20\%), level of anterior portion of mediastinum (5\%) and level of thymus (5\%) [12]. Since the parathyroid gland lacks capsular fixation, gland located in normal anatomic position may also undergo enlargement and get displaced to ectopic location. Large cervical parathyroid adenomas may be sucked into mediastinum due to oesophageal peristalsis or negative intrathoracic pressure.

In our institution, 104 parathyroid neoplasms were reviewed from 2016 to 2019 over a period of 4 years. There were 84 parathyroid adenomas, 10 parathyroid hyperplasia, 6 atypical parathyroid adenomas and 4 carcinomas. Three of the hyperplasia's were ectopically located in retro sternum and left paratracheal regions. Age ranged from 15 to 87 years. There were 72 women and 32 men. Left inferior parathyroid was most common gland involved.

Various preoperative diagnostic tools are available to detect parathyroid adenoma like Ultrasound, Sestamibi scan, CT (Computed Tomography) and MRI (Magnetic resonance imaging) with an accuracy of $57-68 \%$ depending upon the location and size of the lesion [13]. Ultrasound can localize parathyroid adenomas when they are seen in normal location behind the thyroid lobes, however when they are located near the larynx, trachea, para or retropharyngeal space, it is difficult to locate them [13]. 3 dimensional MRI has a sensitivity of $78 \%$ in locating parathyroid adenoma of neck/upper chest which increases to $88 \%$ in the mediastinum which is equivalent to dual phase $99 \mathrm{mTc}$ Sestamibi Scintigraphy [13]. Increased uptake of $99 \mathrm{mmTc}$ Sestamibi in parathyroid glands is due to a) Size b) Amount of blood flow and c) Mitochondrial energy potential [14]. $99 \mathrm{mmTc}$ is first cleared by the thyroid gland and later by parathyroid gland making it is a specific imaging tool for parathyroid adenomas. This is due to dysfunction of P-glycoprotein uptake in parathyroid glands [14]. Hence 99 mTc Sestamibi scan has a diagnostic accuracy of 85$95 \%$ in detecting parathyroid adenoma. Combined 99 mTc Sestamibi scan and CT combination has $100 \%$ sensitivity and $97.4 \%$ positive predictive value for detecting ectopic parathyroid adenoma [14]. In our patient, CT scan of neck and chest with contrast and 99 mTc Sestamibi scans were performed to localize ectopic parathyroid adenomas.

Approach to hypercalcemia includes assessment of serum PTH levels, renal function test, serum calcium and phosphate assay. If primary hyperparathyroidism is confirmed, further tests to locate the PTH producing gland are performed which include ultrasound, sestamibi scan, CT, PETCT (Positron Emission Tomography Computed Tomography), intraoperative PTH assay, etc. to localize the culprit gland. Surgical excision is the treat- ment of choice. Recurrence is very common if supernumerary pathology and MEN syndrome are missed. Thoracoscopic or mediastinal approach may be required for mediastinal parathyroid adenoma excision in $1-2 \%$ cases [6] similar to our case. Parathyroid adenoma should always be handled very gently. Application of an instrument on the adenoma should always be avoided since it can result in spillage of the tumor and recurrence.

\section{Conclusion}

Increased PTH and calcium levels should alert the physician to look for parathyroid lesions in the neck and also elsewhere for ectopic parathyroid lesions. Since the mediastinum is one of the most common locations for ectopic parathyroid adenoma, it should be diligently searched. Combination of imaging techniques is very useful in locating a parathyroid lesion and helps to avoid re explorative surgery for recurrence.

\section{References}

1. Parbatani R, Tinsley GF, Danford MH (1998) Primary hyperparathyroidism presenting as a giant-cell epulis. Oral Surg Oral Med Oral Pathol Oral Radiol Endod 85: 282-284.

2. Kumar S, Bhate K, Pawar V, Kapil Kshirsagar, Vijay Kumar Gupta, et al. (2016) Osteitis fibrosa cystica of jaws as diagnostic criteria for hyperparathyroidism - A case report with review of literature. Int J Med Pharm Case Reports 6: 1-6.

3. Wanzari PV, Chaudhary A, Reddy V, Pratiksha Hada (2011) Oral manifestations established the diagnosis of hyperparathyroidism: A rare case report. Journal of Indian Academy of Oral Medicine and Radiology 23: 155-158.

4. Okuda I, Nakajima Y, Miura D, Maruno H, Kohno T, et al. (2010) Diagnostic localization of ectopic parathyroid lesions: developmental consideration. Jpn J Radiol 28: 707713.

5. Boom RPA, Peterse JL (1986) A patient with a parathyroid carcinoma. Ned Tijdschr Geneeskunde 130: 1117-1120.

6. Hu J, Ngiam KY, Parameswaran R (2015) Mediastinal parathyroid adenomas and their surgical implications. Ann R Coll Surg Engl 97: 259-261.

7. Cakmak H, Tokat AO, Karasu S (2011) Giant mediastinal parathyroid adenoma. Tuberk Toraks 59: 263-265.

8. Wang C (1976) The anatomic basis of parathyroid surgery. Ann Surg 183: 271-275.

9. Sulaiman L, Nilsson IL, Juhlin CC, Felix Haglund, Anders Höög, et al. (2012) Genetic characterization of large parathyroid adenomas. Endocr Relat Cancer 19: 389-407.

10. Bauer W, Federman DD (1962) Hyperparathyroidism epitomized: The case of Captain Charles E. Martell. Metabolism 11: 21-29.

11. Mohebati A, Shaha AR (2012) Anatomy of thyroid and parathyroid glands and neurovascular relations. Clin Anat 25: 19-31.

12. Tardin L, Prats E, Andrés A, Razola P, Deus J, et al. (2011) Ectopic parathyroid adenoma: Scintigraphic detection and radioguided surgery. Rev Esp Med Nucl 30: 19-23.

13. Ott MC, Malthaner RA, Reid R (2001) Intraoperative radioguided thoracoscopic removal of ectopic parathyroid adenoma. Ann Thorac Surg 72: 1758-1760.

14. Hetrakul N, Civelek AC, Stag CA, Udelsman R (2001) In vitro accumulation of technetium-99 $\mathrm{m}$ sestamibi in human parathyroid mitochondria. Surgery 130: 1011-1018. 